\title{
Nutritional biomarker for dietary assessment - a comprehensive review
}

\author{
G. G. C. Kuhnle and Z. He \\ Department of Food and Nutritional Sciences, Whiteknights, University of Reading, RG6 6AP, UK
}

Over the past decades, research in nutritional epidemiology has resulted in a large amount of information on associations between diet and chronic diseases, and provided evidence for public health campaigns. However, nutritional epidemiology relies on accurate dietary information to investigate associations between diet and disease risk, and most dietary assessment instruments rely to some extent on self-reporting which is prone to systematic bias affected by factors such as age, gender, social desirability and approval ${ }^{(1,2)}$. Self-reporting by FFQ for example, is known to result in over-reporting of fruit and vegetable intake, whereas obese people are known to underreport total energy intake, protein and sugar intake. Statistical models developed to accommodate exposure-measurement errors require at least two exposure assessments with unrelated measurement error and this cannot be achieved using methods relying on self-reporting alone, but nutritional biomarkers can provide such an assessment method. Several biomarkers have been developed and are routinely used for dietary assessment, for example, urinary $\mathrm{N}$ for protein intake or plasma vitamin $\mathrm{C}$ and carotenoids as surrogate marker of fruit and vegetable intake. However, more biomarkers are required to improve dietary assessment and thereby information about associations between diet and disease.

We have conducted a systematic literature search to identify publications between 1980 and 2010 describing the development, validation and application of nutritional biomarkers, either in intervention or in observational studies; in total, more than 500 publications were identified.

Most studies focused on well-established biomarkers such as plasma vitamin C and carotenoids - either as individual compounds or total plasma carotenoids - for fruit and vegetable intake, and correlation coefficients between biomarker and intake were found to be between 0.3 and 0.4. Other biomarkers investigated were phenolic compounds, in particular flavonoids, for fruit and vegetable consumption and alcohol metabolites such as ethyl-glucuronide. Notably, many biomarkers were not validated in controlled dietary intervention studies, but by using self-reported data, thus introducing additional bias. Less commonly used biomarkers were isothiocyanates (for brassica vegetables), contaminants such as methyl-mercury and dioxins for fish and alkylresorcinols for whole grain. Although isotope ratio data is commonly used in Archaeology for dietary analysis, only studies have used it for dietary assessment in nutritional epidemiology.

This review provides comprehensive information about nutritional biomarkers developed so far and potential candidate biomarkers to improve future dietary assessment.

1. Hebert JR, Clemow L, Pbert L et al. (1995) Int J Epidemiol 24, 389-398.

2. Hebert JR, Ma Y, Clemow L et al. (1997) Am J Epidemiol 146, 1046-1055. 